\title{
Impact du retrait glaciaire et adaptation du potentiel hydroélectrique dans les Alpes suisses
}

\author{
Stéphane TERRIER ${ }^{1}$, Martin BIERI², Frédéric JORDAN², Anton J. SCHLEISS ${ }^{4}$ \\ 1. Laboratoire de Constructions Hydrauliques (LCH), Ecole Polytechnique Fédérale de Lausanne (EPFL), Station 18, CH-1015 Lausanne, Suisse \\ - e-mail: stephane.terrier@epfl.ch \\ 2. Pöyry Switzerland Ltd., Hardturmstrasse 161, CH-8037 Zürich, Suisse (anciennement LCH, EPFL) - e-mail: martin.bieri@poyry.com \\ 3. LCH, EPFL et e-dric.ch eau énergie environnement ingénieurs conseils, Ch. du Rionzi 54, CH-1052 Le Mont-sur-Lausanne, Suisse - e-mail: \\ fred.jordan@e-dric.ch \\ 4.LCH,EPFL - e-mail: anton.schleiss@epfl.ch
}

\begin{abstract}
RÉSUMÉ. - Le réchauffement climatique est une réalité alarmante et conduit probablement à une augmentation de la pression sur les systèmes socio-économiques. Cependant, dans les régions de haute-montagne, il pourrait induire l'opportunité d'adapter des aménagements hydroélectriques existants, ou de développer de nouveaux projets. Dans les Alpes, le recul des glaciers produit tout d'abord, principalement l'été, une augmentation du volume annuel de fonte selon les caractéristiques du glacier et du bassin versant dans lequel il se trouve. A partir d'une certaine surface du glacier, la perte de surface ne compense plus la hausse de l'ablation et une diminution significative des apports liés aux glaciers doit être considérée pour l'exploitation hydroélectrique. En outre, la diminution des surfaces englacées libère de nouvelles surfaces, dont certaines ont un potentiel pour la construction de nouveaux barrages.

L'opportunité de construire de nouvelles retenues et centrales hydroélectriques juste à l'aval de glaciers en recul est étudiée à l'aide de deux modèles. Le premier (GlabTop) est utilisé pour prévoir la future topographie et géomorphologie sous les glaciers afin de déterminer l'emplacement optimal de nouvelles retenues. Deuxièmement, le modèle hydrologique-hydraulique RS3.0 CLIMATE simule l'évolution du glacier et du débit à l'exutoire des bassins versants ainsi que la production hydroélectrique.

Le cas du haut bassin versant de l'Aar en Suisse est présenté. La possibilité d'y construire un nouveau barrage et une nouvelle centrale est étudiée, incluant une évaluation de la rentabilité. Le résultat donne une base pour évaluer l'opportunité d'investir dans de tels projets pour assurer l'approvisionnement hydroélectrique futur en Suisse et de fournir l'énergie de pointe pour le réseau européen.
\end{abstract}

Mots-clés : nouveau lac, retrait glaciaire, changement climatique, hydroélectricité

\section{Impact of glacier shrinkage and adapted hydropower potential in the Swiss Alps}

\begin{abstract}
Global warming is an alarming reality and likely leads to an increase of multiple pressures on socio-economic systems. However, in high-mountain regions it might also become an opportunity to adapt existing hydropower schemes and to develop new projects to this reality. In the Alps, the melting of glaciers first produces over the near future an increase of the average annual discharge depending on glacier and catchment characteristics, especially during the summer season. Nevertheless after a certain time, significant decrease of runoff related to glacier melting must be considered for hydropower management. Moreover, the shrinking glaciers free new areas, having the potential for the construction of new dams and reservoirs.

The opportunity to build new dams and hydropower plants downstream of retreating glaciers is studied using two models. The first (GlabTop) is used to predict the future topography and geomorphology underneath the melting glaciers, in order to define the optimal locations of the future dams and reservoirs. Secondly, the RS3.0 CLIMATE rainfall-runoff hydrological model computes the glacier evolution, the river discharge at the outlet of the catchment area as well as the hydropower production of the new schemes.

As a case study the Upper Aare River basin in Switzerland is presented. The opportunity of the construction of a new dam and a hydropower plant is studied, including its economic benefit. The result of the case study provides a basis to assess the potential of investing in such projects to ensure the Swiss hydroelectricity production also in future as well as peak energy for the European grid.
\end{abstract}

Key-words: new lake, glacier shrinkage, climate change, hydropower

\section{INTRODUCTION}

Avec le changement climatique, la température moyenne s'élève sur Terre, mais aussi dans les Alpes. Une des conséquences les plus visibles est le fort recul des glaciers [UNEP, 2007][Zemp et al., 2008].
Chaque année, de nouvelles surfaces sont libérées par les glaciers et révèlent une topographie érodée. A certains endroits, des dépressions (ombilics) sont présentes sous la glace et de nouveaux lacs de surface et profondeur variées peuvent apparaitre lorsque le glacier recule. Ils se forment parfois dans une géologie instable et peuvent être retenus par 
une moraine ce qui représente une menace pour la population vivant à l'aval [Clague\& Evans, 2000][Haeberli et al., 2001] [Huggel et al., 2004][Haeberli et al., 2010]. Cependant, ils sont aussi une opportunité pour développer la production hydroélectrique.

Un second impact est la modification du régime hydrologique des rivières alpines. Lorsque les glaciers se réajustent à de nouvelles conditions climatiques, ils peuvent, selon leur taille, leur hypsographie et les caractéristiques de leur bassin versant, produire plus de débit l'été. Ce processus est cependant temporaire puisque chaque glacier finit soit par trouver un état d'équilibre, soit par disparaitre. Par conséquent, non seulement le régime se modifie, mais également le volume des apports. Cela a un impact important sur les aménagements hydroélectriques existants.

L'opportunité de construire de nouveaux barrages et centrales hydroélectriques a été étudiée systématiquement en Suisse dans le cadre du Programme National de Recherche PNR 61 sur la Gestion durable de l'eau, dans le projet intitulé Des lacs comme conséquence de la fonte des glaciers : chances et risques [NELAK, 2013]. En plus du potentiel hydroélectrique, les dangers naturels liés à ces lacs, ainsi que l'aspect touristique et juridique sont traités [Haeberli et al., 2012].

Le cas de l'évolution des glaciers dans le bassin versant de l'Aar est présenté dans le cadre de cet article.

\section{MODÉLISATION}

\section{II.1. Logiciel RS3.0}

\section{II.1.1. Modélisation hydrologique-hydraulique}

Routing System RS3.0 est un logiciel permettant la simulation hydrologique de bassins versants alpins comprenant des aménagements hydrauliques [Garcia et al., 2007]. La prévision hydrologique est effectuée selon le modèle de type conceptuel semi-distribué GSM-SOCONT (Glacer-Snow Melt SOil CONTribution) [Schaefli et al., 2005][Jordan, 2010]. Le bassin versant est divisé en bande d'altitude de $300 \mathrm{~m}$ pour prendre en compte la variation de la température en fonction de l'altitude. Lorsqu'un glacier est présent, une bande glaciaire est ajoutée en plus de la bande d'infiltration dans le sol (Figure 1a).

Le modèle hydrologique permet une interpolation spatiale des précipitations et températures, ainsi que la prise en compte de l'évapotranspiration, de la fonte de neige et de glace, l'infiltration dans le sol, le ruissellement et le routage dans les cours d'eau. L'aspect hydraulique permet la modélisation de prises d'eau, galeries, retenues, déversoirs et turbines/pompes.

\section{II.1.2. Modèle glaciaire}

Le modèle glaciaire simule l'évolution du glacier à long terme ainsi que le débit de fonte. Son objectif premier est de reproduire les débits plutôt que les variations géométriques du glacier.

Pour chaque bande d'altitude, le glacier est modélisé par un parallélépipède rectangle avec une surface $S$ et une hauteur h. Le volume de fonte $\mathrm{V}_{\text {out }}$ dépend d'un coefficient de fonte $\mathrm{A}_{\mathrm{GL}}$, de la température et de la surface de glace (Figure 1b). Si la hauteur de neige $\mathrm{H}_{\mathrm{N}}$ sur le glacier est non-nulle, elle se transforme progressivement en glace $\mathrm{V}_{\mathrm{GL}}$ selon un coefficient de transformation $\mathrm{A}_{\mathrm{GLN}}$ et la hauteur de neige. L'écoulement de glace $\mathrm{V}_{\text {down }}$ d'une bande à la bande inférieure est pris en compte par une vitesse $\mathrm{U}$, la largeur $\mathrm{L}$ et la hauteur $\mathrm{h}$ des bandes. A chaque pas de temps, un bilan $\mathrm{dV}$ est effectué sur les volumes gagnés et perdus, et le résultat est soit ajouté, soit soustrait du volume de glace de la bande. Ces variations sont reportées sur la hauteur et la surface du volume de glace selon un coefficient de forme de vallée. Le calage du débit glaciaire s'effectue avec les paramètres $\mathrm{A}_{\mathrm{GL}}, \mathrm{A}_{\mathrm{GLN}}$ et $\mathrm{U}$.

\section{II.1.3. Modèle de production d'énergie}

Un algorithme est utilisé pour optimiser la production d'énergie dans les centrales. Il cherche à obtenir le plus grand bénéfice en prenant en compte le prix horaire de l'électricité et un niveau cible de remplissage des retenues. Le niveau cible peut varier durant l'année et permet de considérer le stockage saisonnier. Si le niveau dans le réservoir amont est au-dessus de sa cible, le turbinage est facilité pour abaisser le niveau. Au contraire, s'il est trop bas, le a)

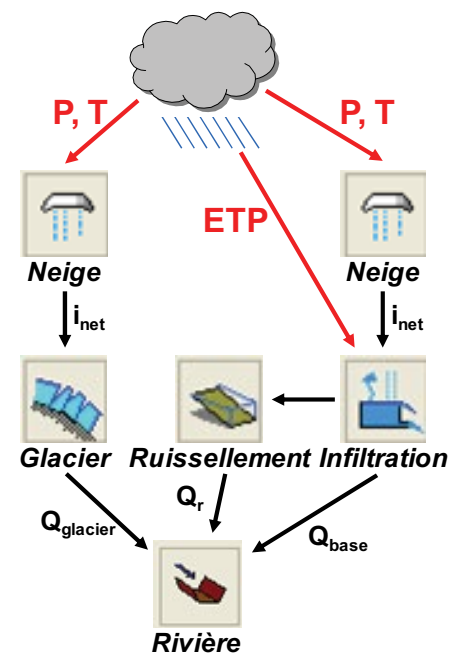

b)

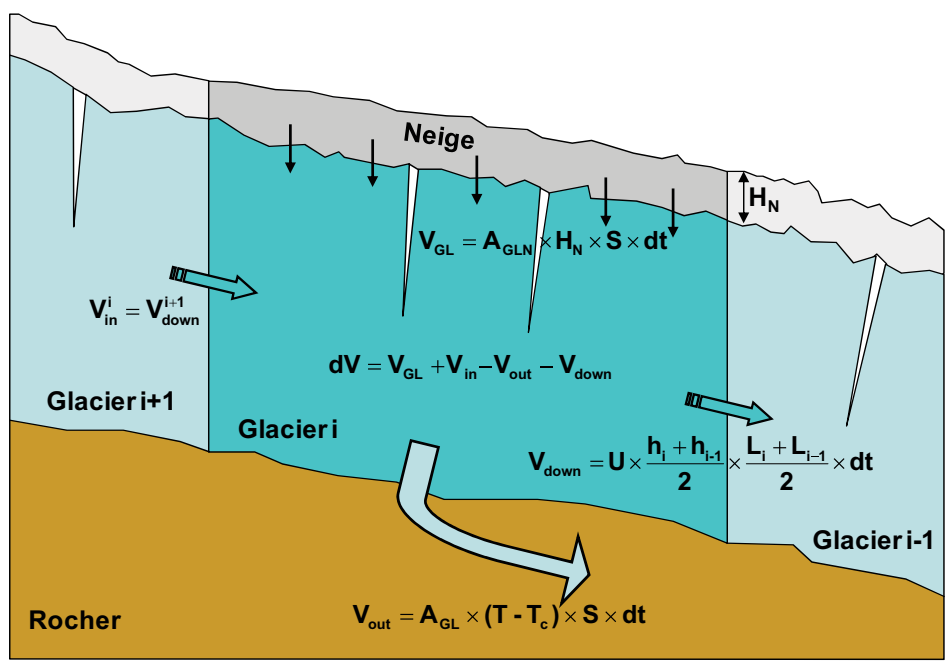

Figure 1 : Logiciel RS3.0 ; a) structure du modèle hydrologique avec l'exemple d'un basin versant avec une bande glaciaire (gauche) et non-glaciaire (droite) ; b) fonctionnement et équations du modèle glaciaire. 
turbinage est diminué. Selon ces paramètres, l'algorithme décide à chaque pas de temps s'il est intéressant de turbiner.

\section{II.2. Scénarios climatiques}

Le climat est en plein changement, et il est nécessaire d'avoir différents scénarios pour une simulation à long terme. Tous les scénarios utilisés se basent sur les données mesurées à plusieurs stations météorologiques à proximité de la zone étudiée entre le 01.01.1980 et le 31.12.2009.

Un premier scénario, nommé ETHZ, est tiré des Swiss Climate Change Scenarios CH2011 [Bosshard et al. 2011] [CH2011, 2011]. Il se base sur le modèle climatique global (GCM) HadCM3Q0 et sur le modèle climatique régional (RCM) CCLM. Il utilise les 30 ans de données de base avec des facteurs correctifs spécifiques à chaque station pour produire les périodes 2021-2050 et 2070-2099. Un coefficient multiplicatif est appliqué aux précipitations et un coefficient additif est appliqué aux températures. En dehors des deux périodes données, une interpolation linéaire est utilisée.

Les autres scénarios admettent une variation linéaire de la température entre 2010 et 2100 et des précipitations inchangées. Les variations étudiées sont de $-2^{\circ}, 0^{\circ}$ (aussi appelé scénario de référence) et $+4^{\circ} \mathrm{C}$.

La construction de tous les scénarios utilise la structure du scénario ETHZ : les années 2021-2050 et 2070-2099 correspondent à 1980-2009, puis 1980-1990 est utilisé pour 2010-2010 et finalement 1991-2009 pour 2051-2069. De cette façon, chaque année de tous les scénarios est construite à partir de la même année de base, ce qui facilite les comparaisons.

\section{II.3. Prix de l'électricité}

L'algorithme de production nécessite une série de prix afin de déterminer quels sont les moments les plus rentables pour turbiner. Les recettes de vente d'énergie sont également obtenues par ce biais.

Pour cette étude, les prix horaires du marché spot EEX du 01.01.2004 au 31.12.2008 ont été choisis. Cette série de 5 ans est répétée sans modification pour obtenir des prix jusqu'en 2100. Ces données ne reflètent pas complètement la réalité du marché électrique en Suisse puisqu'une importante part de la production est vendue par contrat à moyen et long terme. Ces contrats garantissent non seulement une production, mais aussi une puissance à un certain moment et ils ont des prix plus élevés que le marché spot. Ce dernier est néanmoins suffisant pour permettre une optimisation préalable d'un aménagement hydroélectrique.

\section{CAS D'ÉTUDE : LE HAUT BASSIN VERSANT DE L'AAR}

\section{III.1. L'aménagement hydroélectrique KWO}

La zone d'étude se situe dans la partie haute du bassin versant de l'Aar, dans le canton de Berne, en Suisse. Les Forces Motrices d'Oberhasli (KWO) exploitent dans cette région un aménagement hydroélectrique depuis 1932. Ce dernier a été construit et adapté en plusieurs étapes, ce qui en fait aujourd'hui un des aménagements les plus complexes au monde (Figure 2).

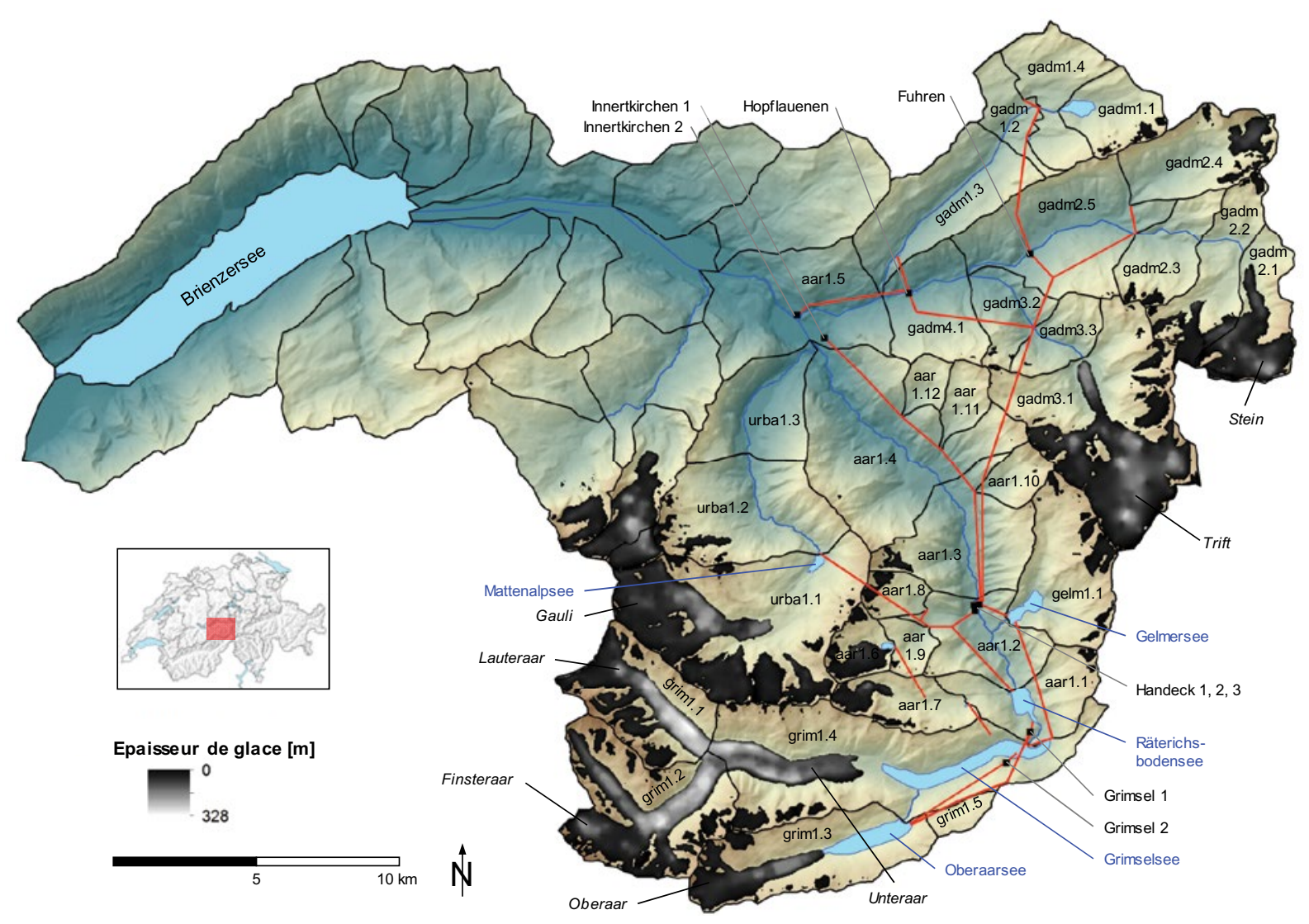

Figure 2 : Bassin versant de l'Aar à l'amont du lac de Brienz et sa couverture glaciaire. La subdivision pour la modélisation hydrologique est montrée avec le nom de chaque sous-bassin. Les traits rouges indiquent les adductions ou galeries en charge actuelles, alors que les carrés noirs donnent l'emplacement des centrales hydroélectriques. 
La surface inclue dans la concession est de $462,5 \mathrm{~km}^{2}$, dont $361 \mathrm{~km}^{2}$ sont exploités pour la force hydraulique. Une partie importante de la surface, $112 \mathrm{~km}^{2}$, est recouverte de glaciers qui produisent d'importants apports durant l'été. Sur les apports annuels moyens de $700 \mathrm{hm}^{3} / \mathrm{an}$, les lacs du Grimsel, d'Oberaar, de Räterichsboden et de Gelmer permettent le stockage de $190 \mathrm{hm}^{3}$.

La production d'énergie annuelle moyenne de $2350 \mathrm{GWh} / \mathrm{an}$ est effectuée dans neuf centrales dont la puissance totale est de $1125 \mathrm{MW}$ en turbinage et $424 \mathrm{MW}$ en pompage.

\section{III.2. Le projet KWO+}

Le projet $\mathrm{KWO}+$, dont la construction a en partie déjà commencée, prévoit d'améliorer le rendement de l'aménagement et d'augmenter la production annuelle. Les quatre parties du projet sont :

- L'augmentation de puissance de $150 \mathrm{MW}$ de la centrale d'Innertkirchen 1, avec la construction d'un nouveau puits blindé et d'un bassin de compensation à l'aval.

- L'augmentation de puissance de $90 \mathrm{MW}$ de la centrale de Handeck 2, avec une nouvelle galerie d'amenée.

- Construction d'une nouvelle centrale de pompageturbinage Grimsel 3 de $600 \mathrm{MW}$ entre les lacs de Räterichsboden et d'Oberaar.

- Surélévation du lac du Grimsel de $23 \mathrm{~m}$, ce qui augmentera son volume de 95 à $170 \mathrm{hm}^{3}$. Cette partie du projet fait actuellement l'objet de plusieurs recours d'organisations écologiques.

Dans cette étude, le projet KWO+ est considéré comme intégralement construit lorsque de nouveaux paliers sont considérés pour les nouveaux lacs.

\section{III.3. Les glaciers et nouveaux lacs potentiels}

Dans le bassin versant exploité pour l'hydroélectricité, une surface de $29,8 \%$ était recouverte par de la glace en 2000 . Les cinq glaciers majeurs sont : Unteraar $12,9 \mathrm{~km}$ de long ( $4^{\mathrm{e}}$ plus long de Suisse, formé par la confluence des glaciers de Finsteraar et Lauteraar), Gauli 6,5 km, Trift 5,7 km, Oberaar 4,9 km et Stein 4,2 km.

L'évaluation de la topographie sous-glaciaire est basée sur un modèle qui estime l'épaisseur de glace en utilisant une loi de flux inverse en combinaison avec une approximation de glace mince [Haeberli \& Hoelzle, 1995][Linsbauer et al., 2012]. Comme le paramètre principal influençant l'épaisseur est la pente de la surface, la méthode étudie la variabilité de l'épaisseur de glace pour des parties du glacier avec des inclinaisons de surface variable dans un schéma spatial explicite. En soustrayant l'épaisseur au MNT (modèle numérique de terrain), le MNT sans glaciers est obtenu. Les dépressions dans la topographie sont de sites potentiels pour de nouveaux lacs. Par la suite, un modèle de retrait des glaciers simplifié [Paul et al., 2007] est utilisé pour classifier les lacs potentiels par leur profondeur moyenne et leur période d'apparition. Même si la probabilité effective qu'un lac se forme est élevée, un certain nombre d'incertitudes liées aux possibilités de drainage demeurent.

A l'aide de cette méthode, plusieurs futurs lacs potentiellement intéressants pour une exploitation hydroélectrique sont détectés dans le bassin versant de l'Aar.

Dans la vallée du glacier de Trift, un lac s'est créé depuis la fin des années 1990 par le retrait du glacier à l'amont d'une gorge. Le glacier s'est entièrement retiré du lac depuis quelques années. Le volume naturel du lac est estimé à $16 \mathrm{hm}^{3}$. Le verrou rocheux formant le lac pourrait permettre la construction d'un barrage d'une hauteur allant jusqu'à $150 \mathrm{~m}$. Avec un volume correspondant de $150 \mathrm{hm}^{3}$, cela permettrait de créer un stockage saisonnier dans le Gadmental qui n'en comporte aucun jusqu'à présent. L'adduction des eaux de la Steinwasser est également souhaitable dans ce cas.

Ultérieurement, deux lacs intéressants se formeront à Gauli permettant de gagner la chute actuellement inexploitée jusqu'à Räterichsboden et d'augmenter la capacité de stockage. Leur utilisation et liaison avec la retenue du Grimsel a été étudiée mais n'est pas présentée. Pour finir, d'autres lacs se formeront sous l'actuel glacier d'Unteraar, mais au vu de leur faible différence d'altitude avec la retenue du Grimsel, ils ne représentent pas un potentiel intéressant et ne sont donc pas montrés.

\section{III.4. Modèle et calage}

Un modèle existant de l'aménagement KWO construit avec l'outil de modélisation Routing System RS3.0 est utilisé comme base [Bieri et al., 2011][Bieri, 2012][Bieri et al., 2013]. Ce modèle est adapté pour tenir compte des nouveaux développements effectués sur le modèle glaciaire et le modèle de production d'énergie.

Pour effectuer le calage des débits, les mesures des apports entrants dans les retenues principales entre 1981 et 2005 sont utilisées. Les apports mesurés sont obtenus à partir de la variation de la mesure de niveau des retenues.

Le Tableau 1 montre les sous-bassins contribuant à chaque mesure et la performance du calage à l'aide du rapport simulé/ mesuré et du coefficient de Nash [Nash \& Sutcliffe, 1970]. Le modèle reproduit globalement bien les débits. La retenue d'Oberaar est fortement influencée par du pompage-turbinage (débits respectifs de 80 et $93 \mathrm{~m}^{3} / \mathrm{s}$ contre $1,77 \mathrm{~m}^{3} / \mathrm{s}$ annuellement pour les apports naturels), ce qui explique un coefficient

Tableau 1 : Liste des sous-bassins inclus à chaque point de calage et indication de la performance de calage par le ratio des volumes et le coefficient de Nash.

\begin{tabular}{|l|l|c|c|}
\hline Endroit mesuré & Bassin versants concernés & Volume & Nash \\
\hline Oberaar & grim1.3 & 0.89 & 0.73 \\
\hline Grimsel & aar1.6, aar1.7, grim1.1, grim1.2, grim1.4, reck2.2 & 1.00 & 0.86 \\
\hline Räterichsboden & aar1.1, aar1.8, aar1.9, urba1.1 & 1.00 & 0.81 \\
\hline Gelmer & gelm1.1 & 1.01 & 0.73 \\
\hline Trift & gadm2.1, gadm2.2, gadm2.3, gadm2.4, gadm3.1, gadm3.3 & 1.01 & 0.89 \\
\hline
\end{tabular}


de Nash inférieur. Finalement, la retenue de Gelmer est également très influencée par l'exploitation.

En parallèle aux débits, les principaux glaciers sont calés sur leur perte d'épaisseur. Un soin particulier a pu être apporté à la modélisation et au calage des glaciers de l'Aar (Oberaar et Unteraar) puisque des mesures de variation d'épaisseur annuelle sont disponibles dans les rapports Die Gletscher der Schweizer Alpen/The Swiss glaciers [Glaciological Commission of the Swiss Academy of Sciences, 1980-2005]. Pour les autres glaciers, des comparaisons entre des cartes topographiques swisstopo récentes et des versions des années 1980 permettent d'estimer la perte d'épaisseur moyenne nécessaire au calage.

\section{RÉSULTATS ET DISCUSSION}

\section{IV.1. Evolution des glaciers}

Le but du modèle est la reproduction des débits et non la simulation du recul des glaciers. Ces résultats donnent donc la tendance pour chaque bande, mais ne permettent pas prédire les caractéristiques géométriques précises du retrait.

La Figure 3 présente comme exemple l'évolution des bandes d'altitude inférieures du glacier d'Unteraar. Les deux scénarios de réchauffement montrent une fonte similaire avec une disparition des bandes formant la langue vers 2070 pour le scénario ETHZ et 2080 pour le scénario $+4^{\circ}$. Le scénario ETHZ a un réchauffement plus important au début du $21^{\text {e }}$ siècle, raison pour laquelle le glacier se retire plus rapidement. Pour le scénario de référence, la fonte est approximativement constante en-dessous de $2500 \mathrm{~m}$. Au-dessus, pour la bande 7 , un ralentissement de la fonte s'observe dans la deuxième moitié du siècle avec une tendance à la stabilisation. Pour un refroidissement, la $3^{\mathrm{e}}$ bande fond régulièrement et perd la moitié de sa hauteur initiale. La $5^{\mathrm{e}}$ bande voit un ralentissement de la fonte vers 2070 , alors que pour la $7^{\mathrm{e}}$ le ralentissement intervient dès 2030 et se poursuit par un accroissement de l'épaisseur de glace. Dans ce cas purement théorique, une stabilisation puis une augmentation du volume total des glaciers se manifestent dans la deuxième moitié du siècle même si les bandes inférieures continuent de fondre. La première bande, au front du glacier, a une faible hauteur initiale et disparait rapidement quel que soit le scénario. Les bandes situées à des altitudes supérieures suivent la même tendance générale que la $7^{\mathrm{e}}$ bande d'altitude et disparaissent avant la fin du siècle pour les deux scénarios de réchauffement.

Les autres glaciers montrent une évolution similaire. La $3^{\text {e }}$ bande ( 2480 m s.m.) du glacier de Gauli disparaît vers 2050 selon le scénario ETHZ, ce qui fera apparaitre le lac de Gauli amont. Les lacs de Gauli aval et Trift sont déjà existants et peuvent être exploités dès à présent.

\section{IV.2. Evolution des apports}

Le changement du volume des apports et du régime hydrologique durant le $21^{\mathrm{e}}$ siècle aura un impact non-négligeable sur la production hydroélectrique et doit être pris en compte dans une adaptation des aménagements.

La Figure 4 montre les apports annuels naturels (sans adductions) dans la retenue du Grimsel. Pour le scénario ETHZ, les apports augmentent légèrement ces 20 prochaines années avant de rapidement diminuer. Pour le scénario de réchauffement $+4^{\circ}$, la tendance actuelle se maintient jusqu'en 2040, puis les apports diminuent. Pour le scénario de référence, le résultat est une diminution régulière, mais modérée. Pour un refroidissement, une diminution plus rapide que pour la référence est observée.

En 2100, les scénarios de refroidissement et de réchauffement donnent des débits très semblables. D'un apport moyen de $193 \mathrm{hm}^{3}$ entre 1982 et 2008, il ne restera que $67 \%$ des apports pour le scénario ETHZ entre 2090 et 2099, contre $81 \%$ pour le scénario de référence.

Pour les hydrogrammes, il y a beaucoup de différences entre les bassins versants étudiés. Elles proviennent principalement de l'altitude et de la présence de glaciers. L'année 2003, bien qu'exceptionnelle par les températures caniculaires durant l'été, montre bien les tendances générales. L'exemple de la retenue du Grimsel est représentatif des apports exploités pour la production d'énergie.

L'hydrogramme pour le scénario ETHZ (Figure 5a) montre de gros changements au cours du $21^{\mathrm{e}}$ siècle. La fonte des neiges au printemps se produit plus tôt et donne des débits plus élevés L'été, la disparition progressive des glaciers réduit considérablement le débit et est l'origine de la réduction des apports annuels. Avec le scénario de référence

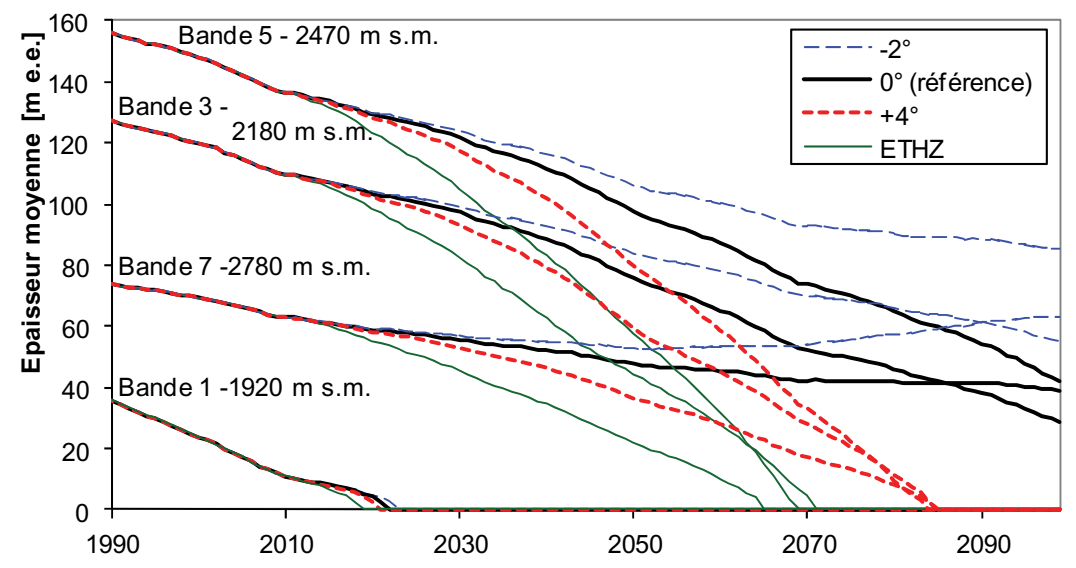

Figure 3 : Evolution de l'épaisseur moyenne de glace selon les scénarios climatiques des bandes d'altitude inférieures du glacier d'Unteraar (bandes 1 et 3) et de Finsteraar (bandes 5 et 7). Les bandes intermédiaires 2, 4 et 6 ne sont pas représentées pour des questions de lisibilité. 


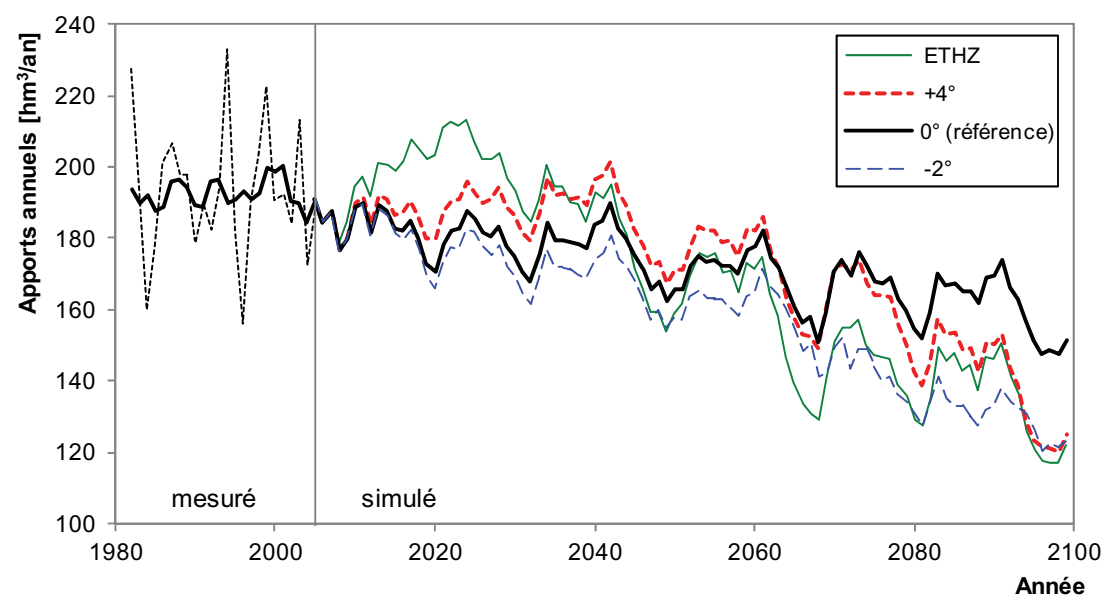

Figure 4 : Evolution du volume annuel des apports naturels de la retenue du Grimsel selon les scénarios climatiques. Les courbes sont lissées en effectuant une moyenne mobile sur 5 ans afin de mieux distinguer les tendances. La courbe traitillée montre la variabilité des valeurs annuelles mesurées.
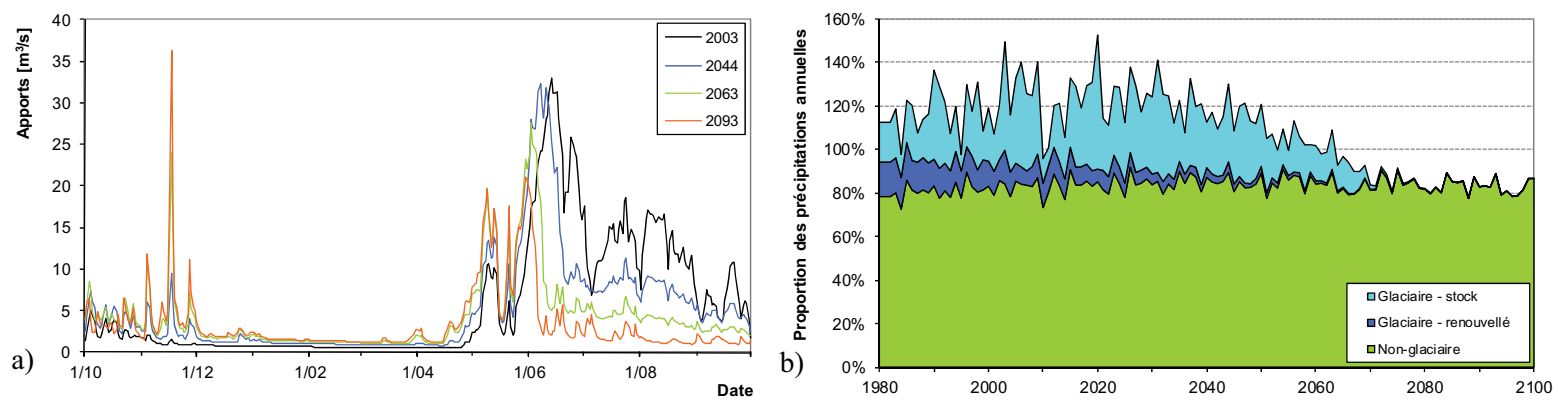

Figure 5 : Pour la retenue du Grimsel et le scénario ETHZ: (a) hydrogramme du débit entrant naturel (les années 2044, 2063 et 2093 sont toutes construites sur la base de l'année 2003); (b) origine des débits.

(non illustré), seule la période de fin juin à septembre subit des modifications suite à la diminution des apports glaciaires liée au recul du glacier.

L'origine des débits pour la retenue du Grimsel est également étudiée. Avec le scénario ETHZ, l'importante fonte du glacier d'Unteraar jusqu'aux environs de 2070 engendre d'importants apports pour les 30 prochaines années. La Figure $5 \mathrm{~b}$ montre que le volume des apports est actuellement plus élevé que celui des précipitations dans le bassin versant. Cette situation n'est évidement pas durable et provient du rééquilibrage des glaciers. La contribution de la perte de masse glaciaire était de $25 \%$ des précipitations ces 30 dernières années et elle sera de $28 \%$ les 30 années qui suivent. Il est probable qu'un volume annuel de fonte aussi important que celui de 2003 survienne durant cette période. La somme des apports de glace renouvelée (le volume de glace fondu durant l'année est compensé par un volume identique de neige transformée en glace) et de débit non-glaciaire diminue au cours du siècle à cause de l'augmentation de l'evapotranspiration qui représente près de $20 \%$ en 2100 .

\section{IV.3. Potentiel hydroélectrique}

De nouveaux aménagements ont été étudiés pour Trift et Gauli. L'analyse énergétique et économique inclut ces deux aménagements, cependant seule la situation de Trift est présentée.

\section{IV.3.1. Projet de Trift}

Trois altitudes différentes de couronnement du barrage ont été étudiées pour le nouveau lac de Trift: 1720, 1760 et $1800 \mathrm{~m} \mathrm{s.m}$. L'idée est de construire un réservoir pour stocker l'eau, produire de l'énergie de pointe et d'avoir la capacité d'exploiter la retenue en env. $1000 \mathrm{~h} / \mathrm{an}$. Les volumes crées par le barrage sont respectivement de 65, 105 et $155 \mathrm{hm} \mathrm{m}^{3}$. Les apports du bassin versant naturel ont été en moyenne de $112 \mathrm{hm}^{3} /$ an entre 1981 et 2005 . L'adduction des eaux de la Steinwasser apporte environ $60 \mathrm{hm}^{3} / \mathrm{an}$.

La variante avec un couronnement à $1760 \mathrm{~m}$ s.m., qui donne un barrage de $115 \mathrm{~m}$ de haut, est la plus intéressante économiquement. Pour le vider en $1000 \mathrm{~h} / \mathrm{an}$ il faut un débit équipé d'environ $40 \mathrm{~m}^{3} / \mathrm{s}$ en considérant l'adduction de Steinwasser. Avec un tel débit, il n'est pas possible d'utiliser ou de lier la nouvelle centrale au système existant et il est choisi de placer une nouvelle centrale directement à Innertkirchen (Figure 6). Avec une chute brute entre 810 et $920 \mathrm{~m}$, la puissance brute est de $440 \mathrm{MW}$. Un bassin de compensation est créé pour réduire le marnage dans l'Aar. Le coût de construction est estimé à 472 mio. EUR 


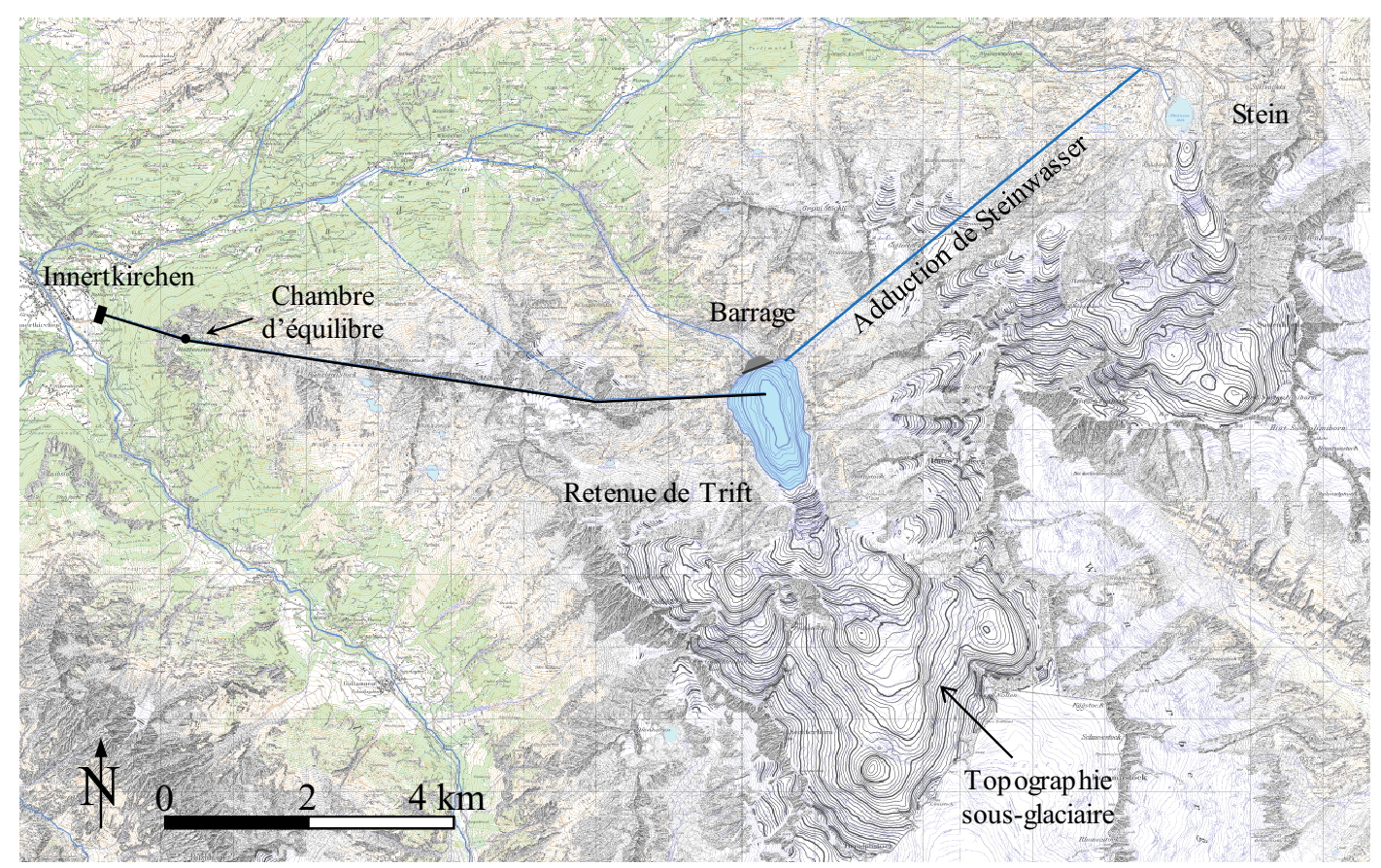

Figure 6 : Plan de l'aménagement de Trift. Reproduit avec l'autorisation de swisstopo (BA110005).

pour une production de $420 \mathrm{GWh} / \mathrm{an}$. Par rapport à l'utilisation actuelle de ces apports, c'est un supplément de $196 \mathrm{GWh} / \mathrm{an}$. Le prix de revient s'élève à $84 \mathrm{EUR} / \mathrm{MWh}$ si l'on considère que les aménagements existants sont amortis et que le nouvel ouvrage peut être rentabilisé par la totalité de la production (pas de dédommagements aux centrales qui perdent de l'eau).

\section{IV.3.2. Analyse énergétique et économique des aménagements existants et futurs}

Les nouveaux paliers de Trift/Innertkirchen 3 et de Gauli sont implémentés dans le modèle RS3.0 de KWO+ afin d'effectuer une comparaison des différents aménagements. L'étude est effectuée sur trois périodes de cinq ans et les résultats sont moyennés pour obtenir des valeurs annuelles. Les trois périodes sont : 1990-95 (comme référence), 2031-36 (avec exploitation des nouveaux lacs) et 2080-85 (fin du siècle avec baisse des apports).
Les données météorologiques de ces années sont toutes basées sur 1990-95, ce qui permet de ne pas avoir de différences provenant d'une variation interannuelle des apports. Toutes les simulations sont faites avec le scénario de réchauffement ETHZ.

La production de chaque centrale est donnée au Tableau 2. La production totale de l'aménagement $\mathrm{KWO}+$ entre 1990-95 et 2031-36 est semblable, alors qu'une perte d'environ $250 \mathrm{GWh} /$ an apparaît entre 2031-36 et 2080-85 due à la diminution des apports.

L'augmentation de la production avec l'aménagement KWO+ par rapport à l'actuel provient seulement de la centrale de pompage-turbinage Grimsel 3, puisqu'il n'y a pas d'exploitation d'un nouveau palier. Par contre, la concentration du temps de turbinage annuel des autres aménagements $\mathrm{KWO}+$ permet de vendre à de meilleurs prix.

Avec l'aménagement incluant les nouveaux lacs, il y a un gain de production par l'exploitation de deux nouveaux

Tableau 2 : Production des différentes centrales existantes en combinaison avec les futurs lacs de Gauli (centrale NL/Gauli) et de Trift (centrale NL/Innertkirchen 3) [GWh/an]. Les abréviations T et P désignent respectivement turbinage et pompage.

\begin{tabular}{|c|c|c|c|c|c|c|c|}
\hline \multirow{2}{*}{ Période Amenagement } & \multicolumn{2}{|c|}{ 1990-1995 } & \multicolumn{3}{|c|}{ 2031-2036 } & \multicolumn{2}{|c|}{ 2080-2085 } \\
\hline & Actuel & KWO+ & Actuel & KWO+ & New Lakes & KWO+ & New Lakes \\
\hline KWO (T) & $2^{\prime} 562$ & 2'338 & $2^{\prime} 536$ & 2'320 & $2^{\prime} 086$ & $2^{\prime} 074$ & 1'920 \\
\hline KWO (P) & $-1^{\prime} 135$ & $-1^{\prime} 036$ & $-1 ' 157$ & $-1^{\prime} 044$ & $-1^{\prime} 068$ & $-1^{\prime} 055$ & $-1^{\prime} 084$ \\
\hline KWO+ / Grimsel $3(\mathrm{~T})$ & & $1 ' 456$ & & $1 ' 509$ & $1 ' 528$ & $1^{\prime} 508$ & $1^{\prime} 524$ \\
\hline KWO+ / Grimsel $3(\mathrm{P})$ & & $-1 ' 782$ & & $-1 ' 887$ & $-1^{\prime} 823$ & $-1^{\prime} 948$ & $-1 ' 895$ \\
\hline NL / Innertkirchen 3 & & & & & 456 & & 365 \\
\hline NL / Gauli & & & & & 31 & & 24 \\
\hline (turbinage) & $2 ' 562$ & 3'794 & $2^{\prime} 536$ & $3^{\prime} 829$ & $4^{\prime} 100$ & 3'582 & 3'834 \\
\hline (pompage) & $-1 ' 135$ & $-2^{\prime} 818$ & $-1 ' 157$ & $-2 ' 931$ & $-2 ' 891$ & $-3^{\prime} 003$ & $-2 ' 979$ \\
\hline
\end{tabular}


Tableau 3 : Recettes de vente d'électricité si toute l'énergie est vendue sur la marché spot actuel [mio EUR].

\begin{tabular}{|c|c|c|c|c|c|c|c|}
\hline \multirow{2}{*}{ Période Amenagement } & \multicolumn{2}{|c|}{$1990-1995$} & \multicolumn{3}{|c|}{ 2031-2036 } & \multicolumn{2}{|c|}{$2080-2085$} \\
\hline & Actuel & KWO+ & Actuel & KWO+ & New Lakes & KWO+ & New Lakes \\
\hline KWO (T) & 161.6 & 154.9 & 160.3 & 152.7 & 142.9 & 135.5 & 128.7 \\
\hline KWO (P) & -37.7 & -35.7 & -38.0 & -35.8 & -37.1 & -35.8 & -37.1 \\
\hline KWO+ / Grimsel $3(\mathrm{~T})$ & & 98.3 & & 100.4 & 101.9 & 99.5 & 100.4 \\
\hline KWO+ / Grimsel $3(\mathrm{P})$ & & -63.5 & & -66.3 & -63.6 & -67.5 & -65.2 \\
\hline NL / Innertkirchen 3 & & & & & 35.6 & & 28.0 \\
\hline NL / Gauli & & & & & 2.5 & & 2.1 \\
\hline Somme & 124.0 & 154.0 & 122.3 & 151.1 & 182.3 & 131.8 & 156.9 \\
\hline
\end{tabular}

paliers. En déduisant les pertes aux centrales existantes, le gain est de $305 \mathrm{GWh} /$ an pour 2031-36 et de $263 \mathrm{GWh} / \mathrm{an}$ en 2080-85 pour Trift. Le gain par le turbinage des eaux de Gauli entre Grimsel et Räterichsboden est difficilement chiffrable à cause de la multitude de centrales pouvant exploiter cette chute, mais est estimée à $60 \mathrm{GWh} / \mathrm{an}$. Les gains de production obtenus permettent de compenser les pertes de production de l'aménagement actuel dues à la disparition des glaciers.

Cette étude se base sur le prix du marché spot. Ce prix ne représente pas complètement la situation du marché électrique. De meilleurs prix peuvent être attendus avec des contrats de vente à l'avance avec puissance garantie, d'autant plus que les Forces Motrices d'Oberhasli disposeraient de $2.3 \mathrm{GW}$ de puissance installée entièrement réglable.

\section{CONCLUSIONS}

Le cas présenté dans cet article s'est concentré sur l'aménagement de KWO, un aménagement d'accumulation typique. La démarche est générale et est applicable à d'autres cas d'étude. L'aménagement des Forces Motrices de Mauvosin [Terrier et al., 2011], ainsi que le glacier du Rhône [Zumofen, 2012] ont ainsi été étudiés.

Quel que soit le scénario climatique, il faut s'attendre à une réduction des apports au cours du $21^{\mathrm{e}}$ siècle. L'hydrogramme sera modifié principalement en été. Ces changements auront un impact sur la production hydroélectrique.

Les pertes de l'aménagement existant suite à la réduction des apports futurs peuvent être partiellement compensées par le développement de nouveaux lacs apparaissant avec le retrait des glaciers. Dans le cas de KWO, deux nouveaux paliers utilisant de nouveaux lacs glaciaires apparus cette dernière décennie sont intéressants. Le premier relie le lac de Gauli à la retenue du Grimsel, tandis que le second, de volume plus important, relie le lac de Trift à Innertkirchen et comprend une nouvelle adduction des eaux de la Steinwasser. L'aménagement des ces deux lacs permet d'augmenter la capacité de stockage pour permettre de produire plus d'énergie de pointe, mais aussi de tirer plus d'énergie des cours d'eau actuellement exploités.

L'étude effectuée dans RS3.0 avec les prix du marché spot a montré qu'il est potentiellement rentable d'exploiter ces nouveaux lacs. Avec le développement des énergies renouvelables comme le solaire ou l'éolien, et le besoin d'énergie réglable pour maitriser la production irrégulière de ces dernières, ces projets de nouveaux lacs ne sont pas seulement économiquement intéressants, mais également indispensables à l'avenir.

\section{REMERCIEMENTS}

Les auteurs remercient particulièrement les Kraftwerke Oberhasli AG (KWO) pour avoir fourni leurs données d'exploitation.

L'étude a été financée par le projet NELAK du Programme National de Recherche PNR 61.

The delta change scenario data were distributed by the Center for Climate Systems Modeling (C2SM).

\section{REFERENCES ET CITATIONS}

BIERI M., SCHLEISS A.J. (2011) - Modelling and analysis of hydropeaking in alpine catchments equipped with complex hydropower schemes. Proceedings of 34th IAHR World Congress, Brisbane, Australia. 2752-2759

BIERI M., JORDAN F., SCHLEISS A.J. (2013) - Bewirtschaftung alpiner Speicherkraftwerke unter Berücksichtigung klimatischer und wirtschaftlicher Veränderungen. WasserWirtschaft. 5 : 36-39

BIERI M. (2012) - Operation of complex hydropower schemes and its impact on the flow regime in the downstream river system under changing scenarios. Communication LCH, Lausanne: EPFL. 52

Bosshard T., Kotlarski S., Ewen T., SchÄr C. (2011) - Spectral representation of the annual cycle in the climate change signal. Hydrology and Earth System Science. 15 : 2777-2788

Clague J.J., Evans S.G. (2000) - A review of catastrophic drainage of moraine-dammed lakes in British Columbia. Quaternary Science Reviews. 19(17-18) : 1763-1783

CH2011 (2011) - Swiss Climate Change Scenarios CH2011. Zurich, Switzerland

Garcia J., Jordan F., Dubois J., Boillat J.-L. (2007) - Routing System II, Modélisation d'écoulements dans des systèmes hydrauliques. Communication LCH, Lausanne: EPFL. 32

Glaciological Commission Of The Swiss Academy Of Sciences (1980-2005) — The Swiss Glaciers: Glaciological report. Zurich: VAW/ETHZ. 102-126

Haeberli W., Clague J.J., Huggel C., KäÄB A. (2010) - Hazards from lakes in high-mountain glacier and permafrost regions: Climate change effects and process interactions. Proceedings XI Reunión Nacional de Geomorphología, Solsona, Spain 
Haeberli W., Hoelzle M. (1995) - Application of inventory data for estimating characteristics of and regional climate-change effects on mountain glaciers: a pilot study with the European Alps. Annals of Glaciology. 21 : 206-212

Haeberli W., KäÄв A., Vonder Mühll D., Teysseire P. (2001) - Prevention of outburst floods from periglacial lakes at Grubengletscher, Valais, Swiss Alps. Journal of Glaciology. 47(156) : 111-122

Haeberli W., Schleiss A.J., Linsbauer A., KünZler M., BüTler M. (2012) - Gletscherschwund und neue Seen in den Schweizer Alpen. Wasser Energie Luft. 104(2) : 93-102

Huggel C., Haeberli W., KaAb A., Bieri D., Richardson S. (2004) - An assessment procedure for glacial hazards in the Swiss Alps. Canadian Geotechnical Journal. 41(6) : 1068-1083

Jordan F., Boillat J.-L., Schleiss A.J. (2010) — Prévision et gestion des crues du Rhône supérieur par l'exploitation optimale des retenues alpines. La Houille Blanche. 5 : 91-102

Linsbauer A., Paul F., Haeberli W. (2012) — Modeling glacier thickness distribution and bed topography over entire mountain ranges with GlabTop: Application of a fast and robust approach. Journal of Geophysical Research. 117(3)

NAsh J.E., Sutcliffe J.V. (1970) — River flow forecasting through conceptual models, part 1 - A discussion of principles. Journal of Hydrology. 10(3) : 282-290
NelaK (2013) - Neue Seen als Folge des Gletscherschwundes im Hochgebirge - Chancen und Risiken. Formation des nouveux lacs suite au recul des glaciers en haute montagne chances et risques. Forschungsbericht NFP 61. Zürich:vdf Hochschulverlag

Paul F., Maisch M., Rothenbühler C., Hoelzle M., Haeberli W. (2007) - Calculation and visualisation of future glacier extent in the Swiss Alps by means of hypsographic modeling. Global and Planetary Change. 55(4) : 343-357

Schaefli B., Hingray B., Niggly M., Musy A. (2005) - A conceptual glacio-hydrological model for high mountainous catchments. Hydrology and earth system sciences. 9 : 95-109

Terrier S., Jordan F., Schleiss A.J., Haeberli W., Huggel C., KÜNZLER M. (2011) - Optimized and adapted hydropower management considering glacier shrinkage scenarios in the Swiss Alps. Proceedings of 79th annual meeting ICOLD, Lucerne, Switzerland. 497-508

UNEP (2007) — Global outlook for ice \& snow. Arendal:UNEP/GRID

Zemp M., Roer I., KäÄв A., Hoelzle M., Paul F., Haeberli W. (2008) - Global glacier changes: facts and figures. Zurich:UNEP/WGMS

ZuMOFEN D. (2012) - Changements climatiques et nouvelles opportunités de production hydroélectriques. Projet de Master, Ecole Polytechnique Fédérale de Lausanne, Suisse 